

\title{
Development of a disposable force- sensing glove for clinicians and demonstration of its force measurements on patients during rehabilitation following anterior cruciate ligament reconstruction surgery
}

\author{
Wei-Chih Wang \\ David R. Linders \\ David J. Nuckley
}




\title{
Development of a disposable force-sensing glove for clinicians and demonstration of its force measurements on patients during rehabilitation following anterior cruciate ligament reconstruction surgery
}

\author{
Wei-Chih Wang, ${ }^{a, b, c, d, *}$ David R. Linders, ${ }^{e}$ and David J. Nuckley ${ }^{f}$ \\ aniversity of Washington, Department of Bioengineering, Seattle, Washington, United States \\ bNational Tsinghua University, Department of Power Mechanical Engineering, Hsinchu City, Taiwan \\ 'National Tsinghua University, Institute of Nanoengineering and Microsystems, Hsinchu City, Taiwan \\ dUniversity of Washington, Department of Mechanical Engineering, Seattle, Washington, United States \\ eUniversity of Washington, Department of Electrical Engineering, Seattle, Washington, United States \\ fUniversity of Minnesota, Department of Orthopaedic Surgery, Minneapolis, Minnesota, United States
}

\begin{abstract}
For many clinicians, their effectiveness is dependent on the magnitude of forces they manually apply to their patients. However, current state-of-the-art care strategies lack quantitative feedback, making it difficult to provide consistent care over time and among multiple clinicians. To provide real-time quantitative feedback to clinicians, we have developed a disposable glove with a force sensor embedded in the fingertips or palm. The sensor is based on the fiber-optic bendloss effect whereby light intensity from an infrared source is attenuated as the fiber is bent between a series of corrugated teeth. The sensor fabricated has a very low profile $(10 \times 7 \times 1 \mathrm{~mm})$ and has demonstrated high sensitivity, accuracy, range, and durability. Force feedback up to $90 \mathrm{~N}$ with an average force threshold at $0.19 \mathrm{~N}$ and average sensor resolution at $0.05 \mathrm{~N}$ has been demonstrated. A preliminary clinical study has also been conducted with anterior cruciate ligament reconstruction patients who show significant range of motion improvement when treated with the force-sensing glove. $\odot 2017$ Society of PhotoOptical Instrumentation Engineers (SPIE) [DOI: 10.1117/1.JBO.22.12.127002]
\end{abstract}

Keywords: force sensor; glove sensor; patch sensor; haptics; force feedback; bendloss; strength measurement; pain threshold; anterior cruciate ligament; anterior cruciate ligament rehabilitation.

Paper 170447R received Jul. 10, 2017; accepted for publication Nov. 27, 2017; published online Dec. 20, 2017.

\section{Introduction}

Greater than $70 \%$ of the U.S. will seek clinical care for musculoskeletal ailments, such as muscle strains, ligament strains, joint replacements, and arthritis, at some point during their lives. ${ }^{1}$ The effectiveness of diagnosis, treatment, and evaluation is dependent on the forces that clinicians apply with their hands. However, current practices lack a tool for the measurement of these forces, leaving much of their intervention subjective.

In diagnosis, treatment, and evaluation, the forces that a clinician applies to his/her patients are not currently quantifiable. These forces are based on previous experience rather than on standard procedures. The physical therapist assigns a strength rating on a 1 to $5+$ scale based on his/her perception of how this patient compares to others evaluated during their practice. Another therapist could assign a different strength rating based on experience or perception on any given day. Pain threshold scoring is similarly qualitative as clinicians diagnose or track progress by feel. The problem is presented when a clinician sees a patient over a period of time and is compelled to repeat diagnoses or treatments due to a lack of quantitative history. This has been known to cause frustration for the patients who

*Address all correspondence to: Wei-Chih Wang, E-mail: abong@uw.edu are repeatedly subjected to painful or routine procedures. This problem is clearly amplified when a patient changes therapists, which is common, especially among athletes. Similarly, a chiropractor's work is largely based on their perception of the forces they apply to their patients. In 1992, a study was conducted by the Stanford Stroke Center that surveyed California chiropractors. Among the 177 respondents, in the preceding 2 years, there were 55 occurrences of stroke following $<24 \mathrm{~h}$ after spinal manipulation. ${ }^{2}$ If these chiropractors knew the forces they were applying to their patients, it is possible these adverse consequences could have been avoided.

Numerous other applications have been identified where manual force is critical but not currently quantified. These include infant CPR training, tool manipulations in surgery, and cardiovascular evaluation. In all applications, the lack of quantitative data prevents consistent and communicable results from being shared that could directly link diagnosis data with treatment protocols and recovery results among the community of musculoskeletal practitioners. Even in cases where some of this data is published, there is no practical way for clinicians to utilize the data while practicing because no tools exist to measure the forces that are applied. ${ }^{3}$ 
At least one study has been conducted to investigate the benefits of concurrent quantitative force feedback in teaching students correct procedures. In 1990, a group of 110 physical therapy students were observed while learning a spine mobilization technique with and without force feedback. The force was measured with a force plate under the table where the patient lay. In Ref. 4, it was recorded that "This additional feedback resulted in immediate improvement in accuracy and consistency of performance of the technique. The improvement was found still to posttest and at follow-up posttest be present one week later." ${ }^{.4}$

Similar improvements have been demonstrated with the use of force sensors during other clinical investigations. ${ }^{5-9}$ Healing times were significantly decreased with concurrent force data and recording capabilities. In all of these cases, it has already been shown that quantitative force feedback and recording are beneficial for training and treatment. However, the methods employed by these investigations are expensive, cumbersome, and not widely applicable. Force plates can measure forces only through the table in the vertical direction. The load cells used in several studies were bulky and did not allow therapists to function normally.

A few research groups have developed force-sensing gloves to directly measure the forces between patient and clinician. A tactile glove was developed by Burdea et al., ${ }^{10}$ which measured grasping forces in virtual reality for rehabilitation activities. The glove uses ultrasonic force sensors that proved to be too sensitive to noise generated environmentally and by the wearer. Another force-sensing glove, based upon conductive ink, was developed by Nikonovas et al., ${ }^{11}$ which was slow to respond and would be unsuitable for dynamic measurements. Also, accuracy was modest, demonstrating up to $6 \%$ error in static measurement with decreasing performance after limited use. A resistor-based force-sensing glove has also been developed to study the grasping forces required for several daily tasks. ${ }^{12}$ However, because of the electromagnetic field generated by individuals, the sensor was not effective for measuring forces between two people. These reviewed force-sensing gloves identify the need for a low-profile, universally applicable sensor that will respond dynamically with good accuracy over a large range. Other gloves have been developed that use strain gauges to measure force but require thick, rigid structures to accurately measure bending correlated with force. ${ }^{13}$ A conductive polymer sensor was also employed in a force-sensing glove, but it was found that the force was accurately measured only at a very small point location and two rigid plates each 1-mm thick had to be applied to distribute force application. ${ }^{14}$

To resolve issues identified in these previous studies, a fiberoptic bendloss force sensor has herein been developed to measure manual force application by the clinician. Optical bendloss sensors have been touted for their low cost, high sensitivity, inertness to electromagnetic interference, durability, and repeatability. Many bendloss sensors have been developed for a variety of applications. In a weighing mechanism, an optical fiber was looped around a beam that was supported on one side by a vertical stand. ${ }^{15}$ A weight applied to the end of the beam bent it proportionally to the mass, bending the fiber and attenuating the light signal from the transmitter diode. The sensor demonstrated a linear response under static conditions. Microbend is another bendloss technique whereby the fiber is perturbed between a series of teeth with small spacing. A review of more than 100 microbend sensors showed that the period of the teeth in a sensor can change its sensitivity by orders of magnitude. ${ }^{16}$
The review also found that the region of safe loading for a fiber is less than one fourth of its break stress. In this region, the life of the sensor may exceed 10 billion cycles. A microbend balance was presented in 2004 that used two plates with sharp angular teeth to engage and bend a plastic optical fiber. ${ }^{17}$ They showed highly linear results and fast response times with a 1.0$\mathrm{mm}$-diameter fiber. One major disadvantage of optical sensors is insertion loss, that is, at every point where two optical components are coupled together significant loss occurs, often requiring high powered emitters. Microbend sensors have been used in the automotive industry, ${ }^{18}$ fuel tank monitoring in aircraft, ${ }^{19}$ and structural stability testing for health care. ${ }^{20}$ Currently, however, there exists no commercially available disposable, low-profile microbend sensor suitable for this application.

\section{Methodology}

Fiber optic bendloss sensors have been shown to have high sensitivity and linearity along a large range of forces or pressures. $^{21,22}$ In this application, a thin optical sensor is embedded in a disposable clinical glove at the fingertips and palm to measure the force that a clinician applies with his hands. The force is transmitted to the display unit [Fig. 1(a)] where it is filtered, calibrated, and displayed. The sensor is based on an optical bendloss technique whereby light is attenuated by spatial perturbations through a series of alternating teeth [Figs. 1(b) and 1(c)]. With this method, the sensor can be thin enough to prevent interference with normal clinical practice while remaining inexpensive. It is also electrically inert to electropotentials between patient and clinician, will not interfere with other clinical instrumentation, and is safe to dispose of after use. In the end product, the optical electronics and wireless module are housed in a wrist cuff to which the disposable glove is connected before use. In the prototype presented here, the wrist cuff is connected to the display unit with a cable instead of a wireless connection, and the optical electronics are housed in the glove instead of on the wrist cuff. The display unit includes calibration hardware and a screen and can be mounted to a wall or secured to a stand in the clinic.

The flexible sensor was designed with soft tissue material properties to conform around the ventral tips of the fingers and palm of the hand while providing force feedback over a wide (clinically relevant) force range. Our sensor involves two thin polymer plates sandwiching a $250-\mu$ m-diameter optical fiber [Fig. 1(b)]. The two plates are semirigid and engineered to induce periodic bending in the fiber at a pitch that maximizes loss and ultimate range. On these plates, a series of corrugated teeth engage the fiber when compressed by outside forces and produce bends that attenuate the light passing through the fiber. ${ }^{21,23,24}$ This attenuation of light is proportional to the amount of bending the fiber experiences. In this way, the force applied can be directly related to the light lost-creating a sensor that is electronically inert and highly sensitive to manual forces applied by the hands. Furthermore, the combination of geometry and optical bendloss characteristics creates a low hysteresis highly repeatable nonlinear profile that provides high sensitivity at low forces and large range at higher forces.

\subsection{Bendloss}

The sensor is based on the bendloss property of optical fiber. As a fiber is bent, some of the light that propagates through is lost across the cladding layer and the light intensity that continues through the fiber is attenuated (Fig. 2). Optical fibers are 
(a)

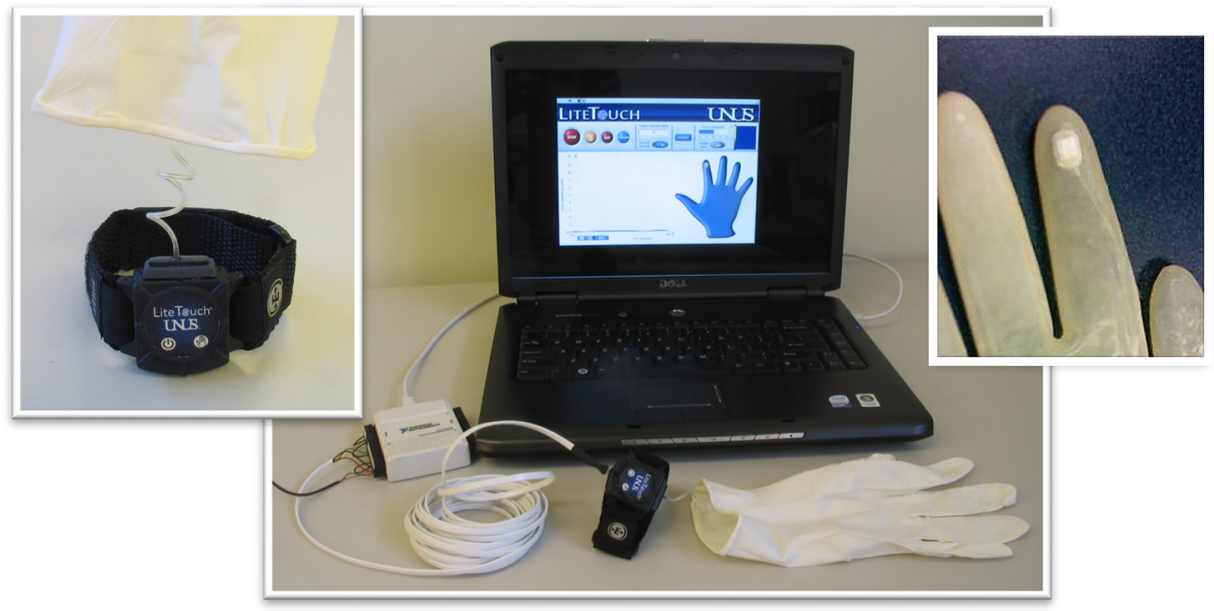

(b)


Fig. 1 (a) Our current force feedback glove system (center): the glove (upper right) embedded with fiber-optic sensors (<1-mm thick) measures applied forces, powered by an LED and detector array in a wrist cuff unit (upper left). Signals collected at the watch are transmitted to a data acquisition system for analysis and shown on the display unit (center). (b) The close-up view of the microbend loss fiber-optic sensor is depicted on the bottom of the figure.



Fig. 2 Bend loss diagram. $R$ is the radius of curvature of the optical fiber at the center axis and $\theta$ is the angle of bending. Some modes from propagating light in an optical fiber will not be reflected inside the fiber, yielding loss in the light intensity measured by a detector.

fabricated with a core layer and a cladding layer surrounding it made from glass or plastic. The index of refraction of the cladding layer is lower than the core such that, under little or no bending, the light undergoes total internal reflection throughout the length of the fiber. When bent past a critical angle, some modes are not reflected off the cladding and escape into the surrounding material.

Tsao and Cheng ${ }^{25}$ have demonstrated, based on experimental results, that total power loss due to bending can be simplified to an exponential function of bending radius and angle ${ }^{26}$

$L_{\text {total }}=\alpha e^{\beta \theta-\gamma r}$,

where $L$ is the total light loss in $\mathrm{dB}, \theta$ is the total angle about which the fiber is bent (360 deg for a full revolution around a mandrel), $r$ is the radius of curvature of the bent fiber, and $\alpha, \beta$, and $\gamma$ are the constants dependent on various conditions, such as fiber type and wavelength. ${ }^{25}$ Each of these constants is determined experimentally. We can then conclude that a sensor that produces bending in an optical fiber around a profile with large radius $r$ and small curvature $\theta$ will yield maximal attenuation.

The correlation between displacement and bending loss can be geometrically calculated. To obtain bending loss as a function of force applied to the sensor requires only the relationship between force and displacement which can be computationally generated with finite-element modeling.

\subsection{Sensor Modeling}

To identify specific geometries and materials that would be sufficient to induce periodic bending in an optical fiber, a finiteelement analysis using ANSYS was performed on a simplified model of the sensor. The top and bottom deformer plates, appearing in turquoise in Fig. 3, were given material properties similar to acrylonitrile butadiene styrene plastic. The fiber was modeled as poly(methyl methacrylate), the same material as the polymer optical fiber ultimately used. The elastomeric filling material has the mechanical properties of polydimethylsiloxane which is similar to the silicone rubber. The sensor was modeled and analyzed in ANSYS with tetrahedral elements. The bottom surface of the bottom deformer was fixed and $120 \mathrm{~N}$ was distributed over the top surface of the sensor.

Based on the results from the optimization, it is found that smaller tooth spacing (pitch) limits the ultimate extent of 

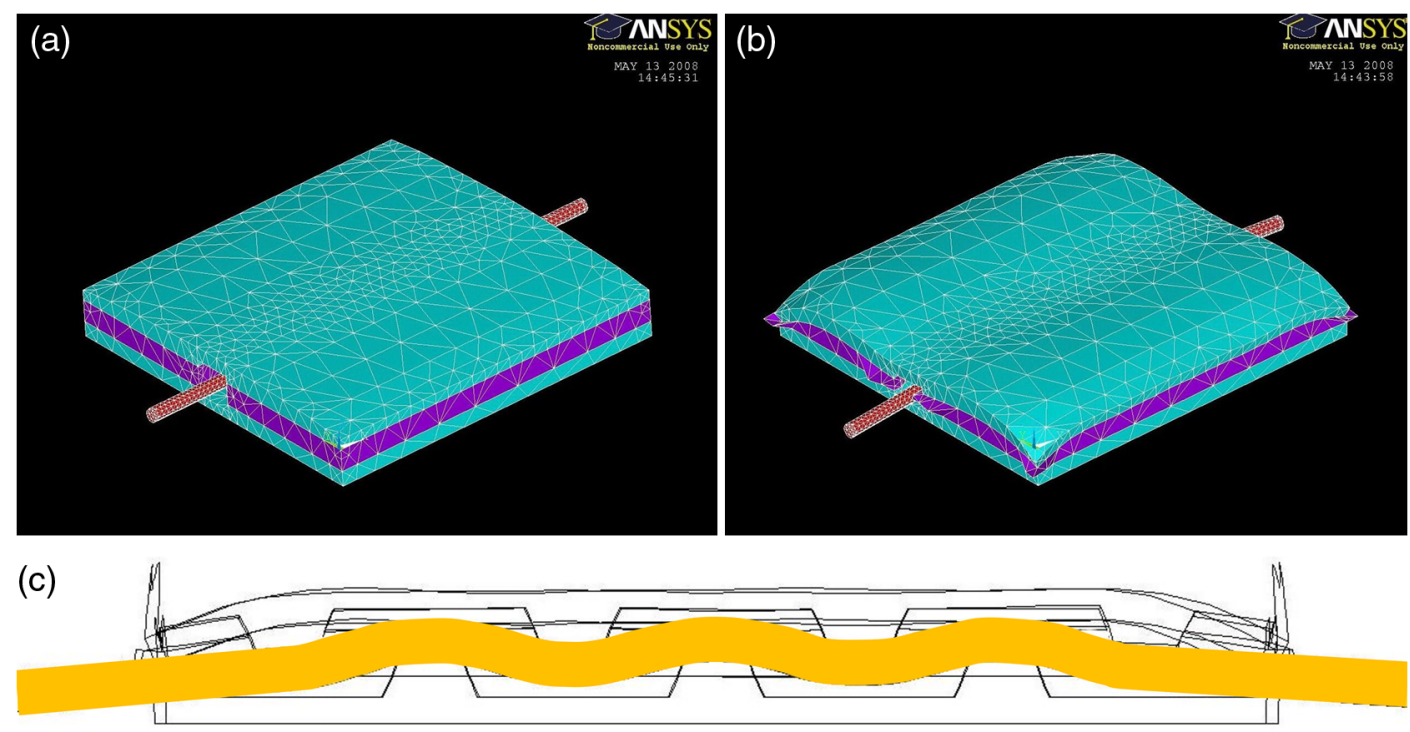

Fig. 3 Results from finite-element analysis performed on a sensor model. The plastic deforming plates are turquoise, the fiber is red, and the elastomeric filling material is purple. (a) and (b) show the sensor before and after loading to $100 \mathrm{~N}$. (c) shows the profile of the bent fiber when loaded.



Fig. 4 Displacement of teeth under quasistatic loading in the finiteelement analysis. The solid line is the best-fit linear regression.

bending while larger pitch induces less bending under equal load. Figure 3(c) was obtained after optimizing the pitch. It shows a cross-sectional view of the sensor $(250-\mu \mathrm{m}$ fiber diameter) with the fiber bent between the teeth under $100 \mathrm{~N}$. At $0.9-\mathrm{mm}$ (seven teeth) pitch, the fiber bends periodically with large deformation.

The simulation was conducted at $20-\mathrm{N}$ increments from 0 to $120 \mathrm{~N}$, and the displacement of the teeth was measured at each step. Displacement increases linearly with quasistatic loading resulting in $0.12-\mathrm{mm}$ displacement at $120-\mathrm{N}$ force as shown in Fig. 4.

These displacements were then applied to a SolidWorks model of the sensor with optimized geometry. Tangential constraints were placed on the fiber, and the bending angle and radius of curvature of the fiber were measured with applied displacements. The bending angles and radii were measured in the model as a function of displacement. The angle is shown to change linearly with applied force while the radius of curvature varies inversely with displacement and force (Fig. 5).

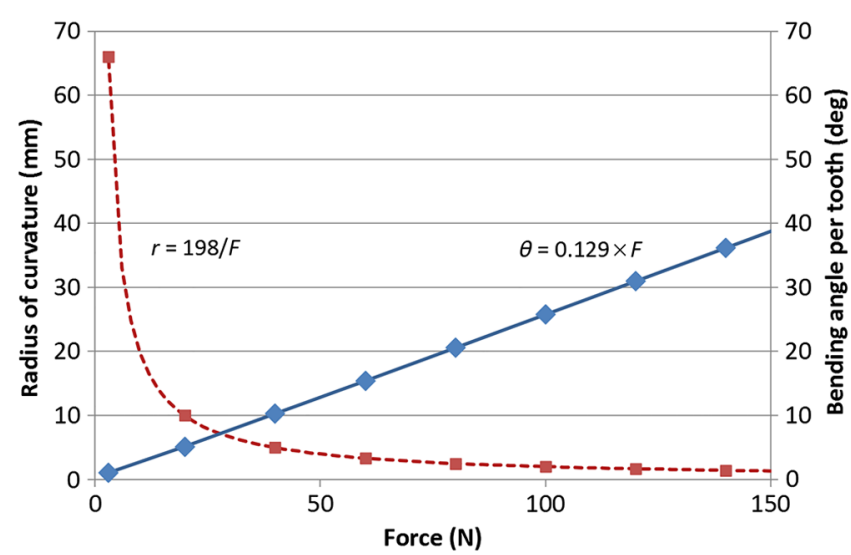

Fig. 5 Computer model of fiber radius of curvature and bending angle as a function of force through displacement data from finite-element analysis.

The plotted angle function represents one bend of which there are seven in the sensor. At $100 \mathrm{~N}$, the fiber is shown to bend at $25 \mathrm{deg}$ with a radius of curvature of $1.96 \mathrm{~mm}$.

The equations relating the radius of curvature $r$ and bending angle $\theta$ as a function of force were then used to generate a theoretical light loss formula based on bending formulas calculated and verified in Ref. 10.

When bending an optical fiber between a series of cylindrical teeth spaced closely enough to approximate successive radial bends in the fiber, bending (and consequently sensitivity) depends on the pitch of the teeth $(p)$, the radius of curvature of the teeth $\left(R_{t}\right)$, and tooth height $\left(H_{t}\right)$. The latter two parameters affect the displacement range attainable by the sensor because of mechanical obstruction. Loss has been empirically measured and shown by earlier Eq. (1). The displacement of the sensor $(d)$ has been shown to be linear with respect to force $(F)$ (Fig. 4). 


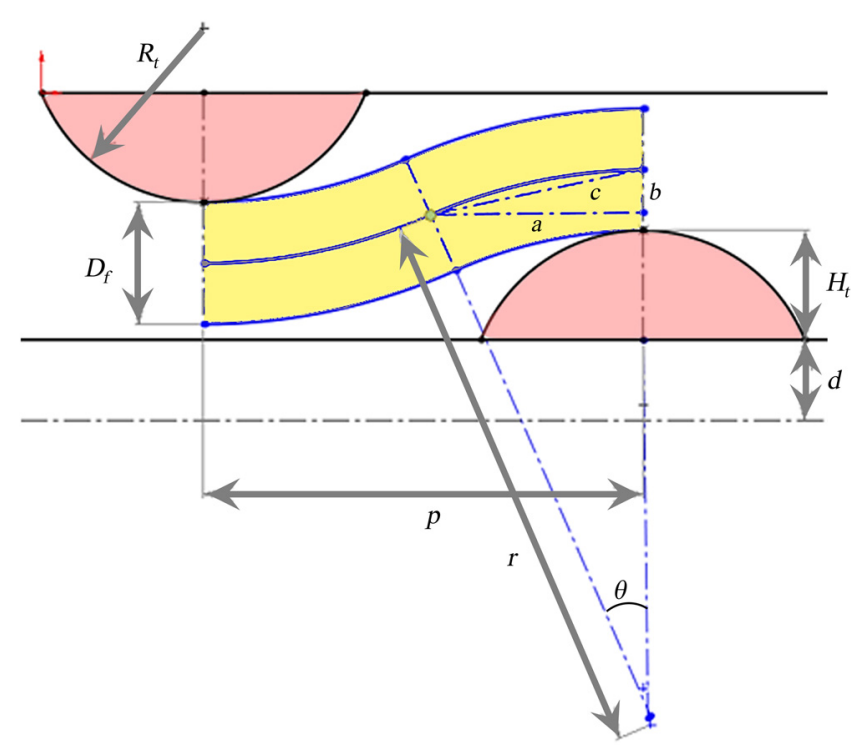

Fig. 6 Diagram of a bending optical fiber between teeth. The bending profile is a function of displacement $(d)$ and tooth pitch $(p)$.

The effect of displacement on bending can be geometrically calculated as demonstrated in Fig. 6. In this calculation, the fiber is assumed to contact both teeth tangentially throughout loading and will be unbent when unloaded $(d=0)$. The bent fiber can be approximated as two arcs tangent to each other and equal in dimension. The average radius of curvature of the fiber is approximated through the axis of the fiber.

Because the fiber bends in two equal portions, segment $b$ is one half of sensor displacement $d$. Similarly, segment $a$ is one half of the pitch $(p)$ because it is the midpoint between the teeth. Therefore, by the Pythagorean theorem

$c^{2}=a^{2}+b^{2}=(p / 2)^{2}+(d / 2)^{2}$.

By law of cosines

$\cos \theta=\frac{2 r^{2}-c^{2}}{2 r^{2}}$

which, after substitution for $c^{2}$ and reducing, becomes

$\cos \theta=1-\frac{p^{2}+d^{2}}{8 r^{2}}$

To obtain a system of two equations to solve for $\theta$ and $r$, we can define the trigonometric relationship

$\cos \theta=\frac{r-d / 2}{r}$

Solving the system gives

$r=\frac{p^{2}-d^{2}}{4 d}$

and

$\theta=\cos ^{-1}\left(1-\frac{2 d^{2}}{p^{2}-d^{2}}\right)$.

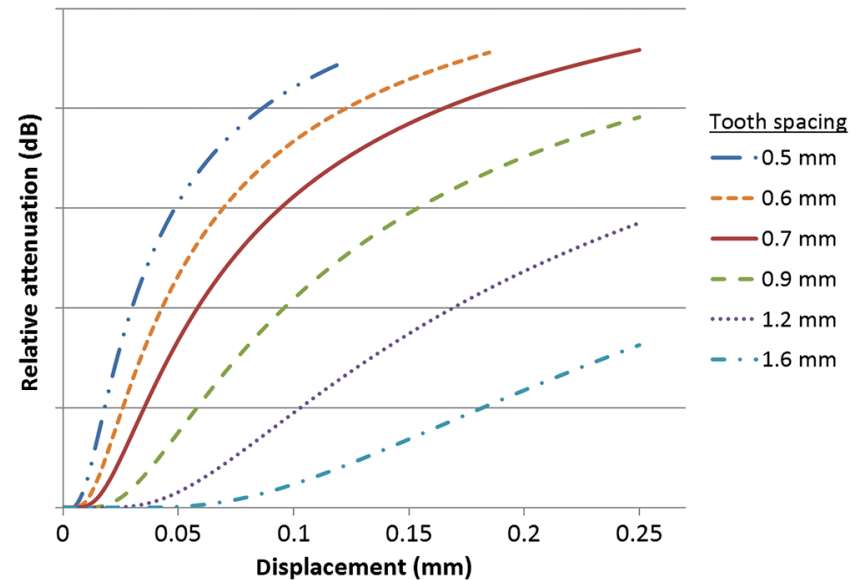

Fig. 7 Mathematical model of relative attenuation as a function of displacement and pitch due to radius of curvature only. With 5- and 6-mm pitch, the mechanical limit of bending is reached before the ultimate sensor compression limit.

The formula for $r$ can be approximated as a reciprocal function $(r=0.2 / d)$ when $d$ is $<0.25 \mathrm{~mm}$ and the formula for $\theta$ can be approximated as a linear function $(\theta=0.13 \times d)$ with respect to displacement in the same region.

These mathematical models validate the finite-element analysis performed with the same geometry. These formulas can also be inserted into the simplified bendloss formula to estimate the relationship between attenuation and displacement. The relationship between attenuation and displacement is dependent on the coefficients of each factor (angle and radius) in Eq. (1) and thus the radius and angle components cannot be easily combined analytically to obtain a loss formula. Other theoretical formulas have been demonstrated to calculate these correction factors.

The relative attenuation due to decreasing the radius of curvature by loading is found to be

$L_{r}=\alpha e^{-\gamma r}=\alpha e^{-\gamma * \frac{p^{2}-d^{2}}{4 d}}$.

As tooth pitch increases, attenuation decreases until the mechanical limit is reached (Fig. 7). In a sensor with tooth pitch of $0.5 \mathrm{~mm}$, height of $0.4 \mathrm{~mm}$, with radius of curvature of $0.25 \mathrm{~mm}$, and a fiber of diameter $0.25 \mathrm{~mm}$, the bending limit is reached at $0.12-\mathrm{mm}$ displacement although $0.25-\mathrm{mm}$ displacement can be reached with larger pitch. In this arrangement, bending sensitivity is highest with the smallest pitch $(0.5 \mathrm{~mm})$ but overall range is optimized with a pitch of $0.7 \mathrm{~mm}$. Sensitivity to bending is initially poor at low displacement, creating a toe region at the beginning of the loading curve. As displacement increases, attenuation asymptotes and the region of highest sensitivity are in the middle.

Relative attenuation due to increasing the angle of bending is given by

$L_{\theta}=\alpha e^{\beta \theta}=\alpha e^{\beta \times \cos ^{-1}\left(1-\frac{2 d^{2}}{p^{2}-d^{2}}\right)}$.

Attenuation is most severe at lower pitches, but the mechanical limit of the sensor is also demonstrated here. Sensitivity to bending is most profound with the smallest pitch $(0.5 \mathrm{~mm})$ but has the highest ultimate range when the teeth are spaced $0.7 \mathrm{~mm}$ 


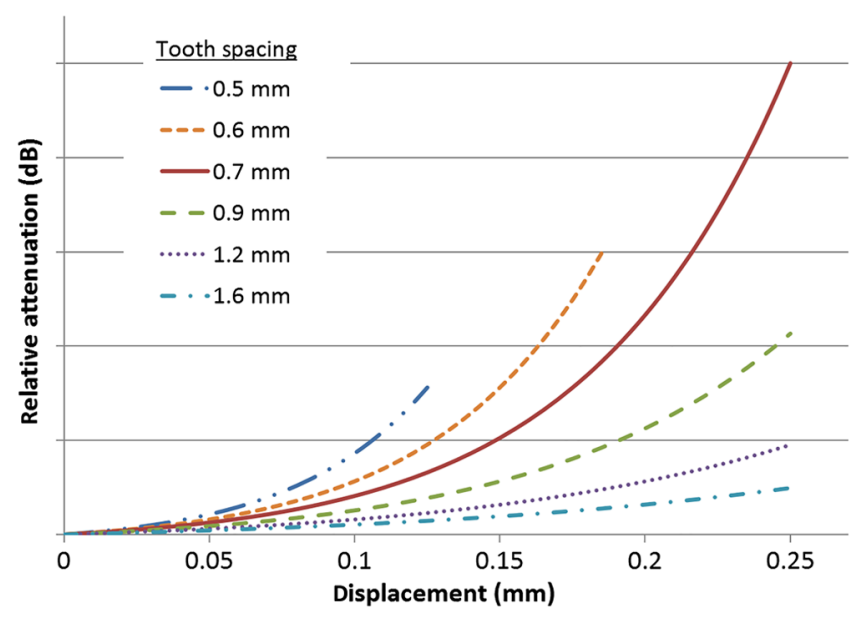

Fig. 8 Mathematical model of relative attenuation as a function of displacement and pitch due to bending angle only. With 5- and 6-mm pitch, the mechanical limit of bending is reached before the ultimate sensor compression limit.

apart (Fig. 8). Sensitivity to bending is again lowest at low displacements.

The total loss can be combined from Eqs. (8) and (9)

$L_{T}=\alpha e^{\beta \theta-\gamma r}=\alpha e^{\beta \times \cos ^{-1}\left(1-\frac{2 d^{2}}{p^{2}-d^{2}}\right)-\gamma * \frac{p^{2}-d^{2}}{4 d}}$.

Using the tooth geometry optimized from finite-element analysis and the theoretical calculations, the sensor was modeled in SolidWorks. To prevent damaging the fiber with severe bending at a low pitch, the teeth were spaced $0.9 \mathrm{~mm}$ apart [Fig. 3(c)]. The teeth were given radii of curvature of $0.4 \mathrm{~mm}$ with a height of $0.25 \mathrm{~mm}$. The top deforming plate contains four teeth and the bottom contains three teeth with a groove on each side for aligning the fiber along the center of the teeth [Fig. 3(c)]. When assembled, the clearance between top and bottom teeth is $0.25 \mathrm{~mm}$, the diameter of the fiber. Two lips were included on the lateral edges of the top deforming plate [Fig. 1(b)] to align the two plastic parts. The top and bottom surfaces of the assembled sensor are contoured slightly to eliminate the feel of edges when mounted on the fingertip. The sensor's assembled footprint is $10 \times 7 \mathrm{~mm}$ and its height is $1.0 \mathrm{~mm}$ [Fig. 1(b)]. The slightly curved contours of the top and bottom surfaces reinforce the applicator plates so that the fiber can bend normally when a load is applied (see Fig. 11). The sensor is also made naturally to respond to a force that's added to a point since the light attenuation is proportional to the total amount of bend occurring in the entire fiber and independent of individual bends in teeth.

\section{Sensor Fabrication}

\subsection{Optical Sensor Assmebly}

Molds were drawn (Fig. 9) that were used to reproduce the sensor in two-part plastic (Smooth-On 385) three sensors at a time. Holes in the corners were included to place disk magnets for mold alignment. The blocks were printed on a three-dimensional printer with $0.1-\mathrm{mm}$ layer thickness in a rigid polymer (Vero Blue from Red Eye RPM). To cast the top and bottom deforming plates, the liquid plastic components were mixed in equal amounts and poured into the cavities on the molds. After partially curing to a sticky gel-like composition, the two halves of the molds were joined magnetically then clamped to press out excess plastic material. After curing for at least $1 \mathrm{~h}$, the sensor parts were removed and trimmed. Mold release was sprayed onto the molds before pouring the plastic to facilitate easy removal.

The optical fiber was cleaved using a razor blade pressed down orthogonally to the fiber. To assemble the sensor, the fiber was then centered in the groove of the bottom plastic deformer. An excess of silicone rubber sealant was applied to the elastomer cavity and the top deforming plate aligned and depressed down, pressing the excess elastomer out the sides. In this way, the whole cavity is filled with the elastomer material, securing the fiber on both ends and holding the deforming plates together adhesively.

\subsection{Optics Integration and Embedding}

The signal is generated by transmitting light through the fiber and measuring the relative intensity after it passes through the fiber between the applicators. An infrared (centered at $940 \mathrm{~nm}$ ) light-emitting diode (LED) light source was used to limit



Fig. 9 CAD drawing of the molding blocks printed for sensor fabrication. 




Fig. 10 Block diagram of signal flow from LED to display. Lookup table is generated by presession calibration.

ambient light noise in the signal. A 3-mm LED used from LiteOn Technology Corporation (LTE-4206) has a radiant intensity of $2.71 \mathrm{~mW}$ at $20 \mathrm{~mA}$. The photodetector paired with this light source was another T-1 packaged 940-nm peak phototransistor (LTR-4206E). The dome on the LED focuses the light into the front $20 \mathrm{deg}$ and the phototransistor also has a half angle of $20 \mathrm{deg}$. The LED supplies light to two parallel fibers per sensor. One fiber passes through the sensor and the other serves as a macrobend correction signal that eliminates the noise generated when the finger or wrist is bent. To couple the fiber to the optical components, the cleaved end of the fiber was inserted into a 5-mm length of $0.6-\mathrm{mm}$ outside diameter wire jacket. This was secured to the LEDs and phototransistor with shrink wrap tubing and epoxied together. The optical components were wired to a four-channel plug (LED supply, ground, and the two phototransistor output channels) that connected to the custom circuit board housed in the wrist cuff. Another four-channel plug was connected to the other side of the printed circuit board (PCB) to be connected to the display unit. The PCB contained simple circuitry to indicate power connection, glove connection, drive the LED, and collect the phototransistor signal to send to the display computer.

To embed the fibers, optical components, and sensor within the glove, an off-the-shelf clinical glove was turned inside-out and inflated and the components were secured with small strips of elastic tape from $3 \mathrm{M}$. The fibers were secured along the back of the hand and along the sides of the pointer finger, crossing over the ball of the fingertip where the sensor was placed (Fig. 1). In this way, the fibers would not be stretched when the finger was bent. The macrobend correction fiber was secured to the back of the finger instead of the fingertip to avoid being crushed. The optical components were taped to the glove on the back of the hand with the connection wires extending out past the wrist for connecting to the wrist cuff. The glove was then turned back inside-out so the fibers, sensor, and optical components were housed inside.

\subsection{Data Acquisition and Testing}

A custom LabVIEW interface was developed to display and record the force measured by the sensor. The sensor requires calibration before every use because of variability in fabrication and variability among users' fingertips. Thus, the first process is to apply the expected maximal load to an external compression load cell (Measurement Specialties FC22) also connected to the display unit. This generates a calibration curve that correlates force and voltage from the phototransistors (the difference between the sensor and macrobend correction channels), which is proportional to the light attenuation. The calibration curve is verified to be monotonically increasing so that every voltage input has exactly one force output and is subsequently saved in a lookup table. The voltage inputs are acquired at a $20-\mathrm{Hz}$ sampling rate, subtracted, and linearly interpolated in the lookup table to calculate the force applied across the sensor (Fig. 10). A moving average filter is applied with variable width that is automatically adjusted with periodic noise measurements on the signal.

Several properties of the sensor are clinically relevant and were tested manually. Force threshold here is defined as the minimum force applied to the sensor that can be detected in the display unit and is important for delicate measurements, such as pulse readings for cardiovascular practices. Range is the maximum force for which a monotonic calibration curve is still obtained. Threshold and range were measured with manual application of the sensor onto the calibration load cell up to its maximum force at $\sim 5 \mathrm{~N} / \mathrm{s}$. Resolution is the smallest difference between two forces that can be differentiated by the sensor and was calculated from the analog-to-digital conversion over the range of input voltages from the phototransistors. Accuracy and stability were observed when loaded up to $24.8 \mathrm{~N}$ in displacement control and held for $30 \mathrm{~s}$. Resolution and accuracy were measured on a linear vertical loading stage (UTM1000P.1, Newport Corporation) controlled by a universal motion controller/driver (ESP300, Newport Corporation). Noise due to finger bending and hand movement was also measured.

\section{Results and Discussion}

\subsection{Numerical Model}

The finite-element analysis revealed that the deformation of the sensor along the $z$-axis is linear with respect to the load, deforming $0.1 \mathrm{~mm}$ at $100 \mathrm{~N}$ (Fig. 4). Under this load, the fiber bends along a periodic path in seven corrugations as shown in Fig. 11(b). The angle and radius of curvature about which the fiber was bent were also measured. The total angle around all seven bends increases linearly with deformation and the radius of curvature decreases as $1 / r$. When substituted into the loss formula from Eq. (1) and rearranged for measuring the voltage loss due to perturbation, the loss equation can be described as

$\Delta V=10^{\alpha e^{\beta F-\gamma / F}}-1$

where $\Delta V$ is the voltage difference, $F$ is force applied, and $\alpha, \beta$, and $\gamma$ are the constants.

Figure 11 demonstrates a qualitative validation of the sensor and agreement between the fabricated sensors and finite-element models. Figure 11(a) shows the periodic deformation of the fiber when compressed in a sensor without elastomeric filling material (this plastic deformation of the fiber is eliminated when the sensor is filled with the elastomeric material described previously). Figure 11(b) shows the deformation of a sensor 


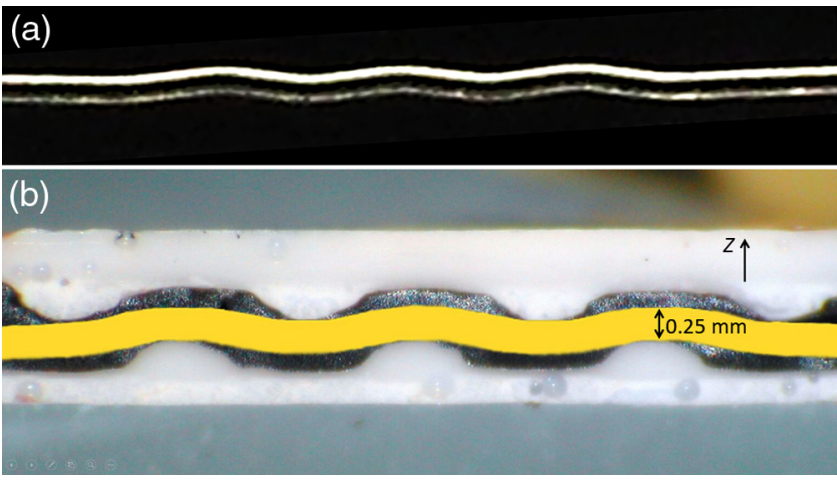

Fig. 11 Quantitative validation of fiber and sensor deformation: (a) deformation of a fiber by the sensor and (b) cross-section cut of the sensor showing teeth and fiber bending profile from finiteelement analysis (yellow).

under load and the resulting cross section of the teeth. The yellow fiber profile was generated with the finite-element model and overlaid onto the photo for shape validation. Here, it shows that the slightly curved contours of the top and bottom surfaces reinforce the applicator plates so that the fiber can bend normally when a load is applied (see Fig. 11). The sensor is also made naturally to respond to a force that's added to a point since the light attenuation is proportional to the total amount of bend occurring in the entire fiber and independent of each individual bend in teeth.

\subsection{Sensor Calibration}

The sensor, when assembled, measures $1.0-\mathrm{mm}$ thick with a footprint of $7 \times 10 \mathrm{~mm}$ [Fig. 1(b)]. The top applicator interlocks with the bottom surface, holding the fiber securely and aligning the teeth correctly. The contours of the top and bottom surfaces allow the sensor to fit comfortably on the fingertip or palm. Even when large forces are applied to the sensor, it does not cause discomfort to clinician or patient.

When calibrated up to $68 \mathrm{~N}$ (15 lbs), the sensor demonstrated strong agreement with expected results based on Eq. (11) from finite-element analysis and typical optical fiber bending response as shown in Fig. 12. The corresponding 1.6-V voltage change shows relatively strong signal transduction. This is

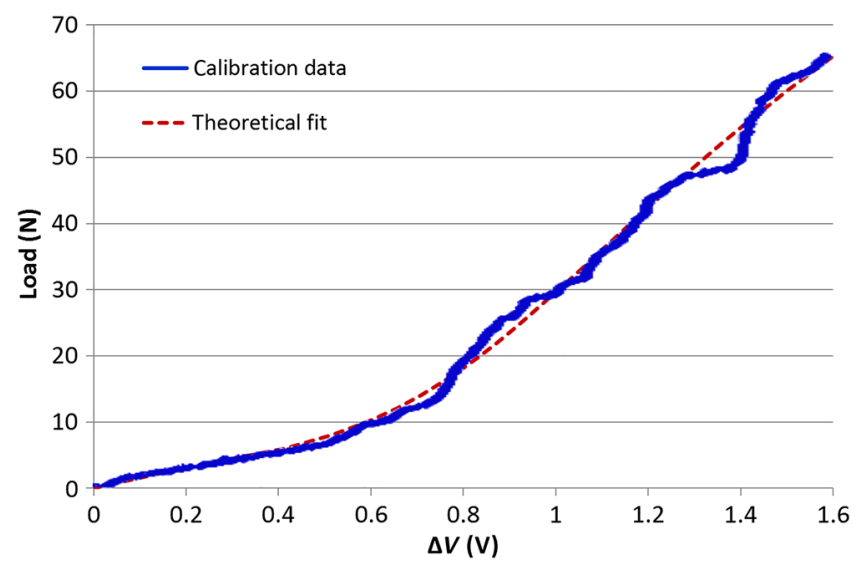

Fig. 12 A typical calibration curve with the theoretical model demonstrating good agreement. The coefficients for the theoretical fit for this specific calibration curve are $\alpha=0.28, \beta=0.0070$, and $\gamma=3.96$. further enhanced by the fact that the LED, phototransistor, and wireless electronics onboard the wrist cuff require fairly small power $(83.5 \mathrm{~mW})$ to operate.

\subsection{Sensor Performance}

Three prototype gloves were tested briefly before being evaluated by clinicians in a physical therapy setting. Initial results have demonstrated that loads up to $90 \mathrm{~N}$ can be applied to the sensor while obtaining a monotonic load-loss curve, that is, a functional calibration curve is generated for measuring force through the sensor. Loads above $90 \mathrm{~N}$ on a fingertip sensor have been difficult to obtain because of a lack of finger strength. Average force threshold has been measured at $0.19 \mathrm{~N}$ and average sensor resolution is $0.05 \mathrm{~N}$.

The whole glove system proved to provide a stable force measurement over time and with tolerable noise in the signal. With no movement, the signal-to-noise ratio (SNR) of the system has averaged $56 \mathrm{~dB}$. When the glove was rolled from the fingertip to measure finger bending noise, the SNR decreased to $20 \mathrm{~dB}$, but displayed only $2.7 \mathrm{~N}(0.6 \mathrm{lbs})$ increase in the force measured. Environmental light had little effect on the force measurement with an average SNR of $33 \mathrm{~dB}$, contributing to a maximum force measurement artifact of $0.6 \mathrm{~N}(0.13 \mathrm{lbs})$.

The sensor's creep is most apparent when held in displacement control for prolonged periods. Figure 13 shows the output force measurement of the sensor and the actual force applied over a 60 -s period. The sensor was initially loaded to $\sim 23 \mathrm{lbs}$ $(104.3 \mathrm{~N})$ and subsequently held in displacement control. Because the sensor output is technically a measure of sensor deformation as it relates to bending, the sensor's measurement varies little over time. The creep in the elastomer is evidenced by the decay in actual force measured by the load cell in series. In $25 \mathrm{~s}$, the actual force decayed from 23 to $18 \mathrm{lbs}(104.3$ to $81.6 \mathrm{~N})$ while the sensor output remained at $22 \mathrm{lbs}(99.8 \mathrm{~N})$. Steady state was achieved after $25 \mathrm{~s}$ with a difference of $4 \mathrm{lbs}(18.1 \mathrm{~N})$ between sensor and load cell outputs.

After holding for $60 \mathrm{~s}$, the load was rapidly removed, and the sensor's relaxation back to $0 \mathrm{lbs}$ was measured (Fig. 14). The sensor's force measurement relaxed to $2 \mathrm{lbs}(9.07 \mathrm{~N})$ within $0.05 \mathrm{~s}$ and to $1 \mathrm{lb}(4.53 \mathrm{~N})$ within $0.45 \mathrm{~s}$. To reach $0.5 \mathrm{lbs}$ $(2.27 \mathrm{~N})$ required an additional $1.5 \mathrm{~s}$ and after $10 \mathrm{~s}$, the sensor's measurement was below $0.2 \mathrm{lbs}(0.91 \mathrm{~N})$. This graph shows the sensor system held in displacement control, that is, the sensor was compressed to a certain displacement and then held for $\sim 60 \mathrm{~s}$ while the drift in the sensor output was observed. Since the bendloss sensor is activated by displacement of the

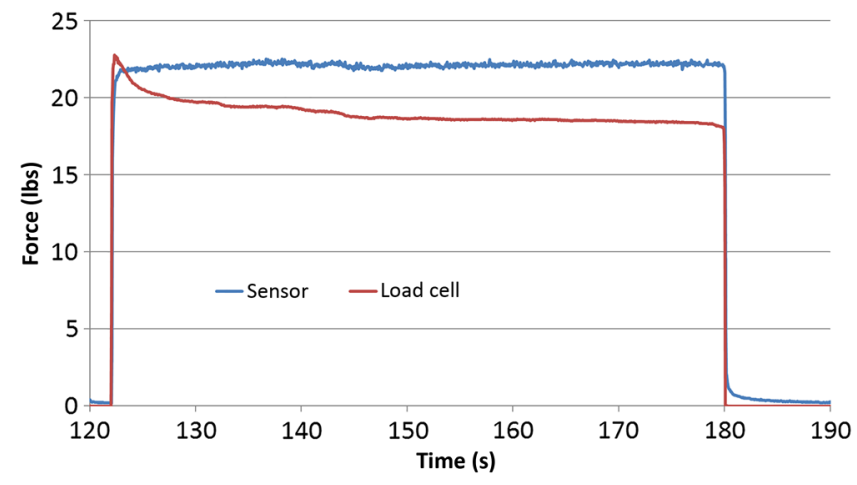

Fig. 13 Sensor creep under displacement control when held for $60 \mathrm{~s}$. 




Fig. 14 Stress relaxation after 60-s $22 \mathrm{lb}$. load.

top and bottom teeth, the sensor value does not drift over time when held at a constant displacement. However, the elastomeric material in the sensor does relax over time and thus exerts less force on the load cell adjacent to the sensor. Therefore, the load cell experiences a significant relaxation over the same 60-s period. The important thing here is to acknowledge that there is a good amount of hysteresis involved that achieves steady state after holding for about $25 \mathrm{~s}$, but with a $20 \%$ change between measured and actual force. However, the sensor comes back to baseline very quickly (within $0.5 \mathrm{lbs}$ of 0 around $1.5 \mathrm{~s}$ after removing the force).

Each sensor was then loaded in displacement control to one half of its range in the same fatigue profile described previously (10 3-s steps, 10 1-s steps, and 10 1/2-s steps) and as shown in Fig. 15. A custom peak finder algorithm was applied to the load cell and sensor time history profiles, and the difference between each peak was measured. The average peak difference between sensor and load cell for 3 -s steps was $0.2 \pm 0.1,0.9 \pm 0.3$ for 1 -s steps, and $1.1 \pm 0.7$ for $1 / 2$-s steps. Latency between sensor and load cell to peak was found to be $0.1 \mathrm{~s}$ and relaxation to $0 \mathrm{lbs}$ occurred within the length of the pulse at each rate. Thus, we can say that the sensor is dynamically responsive to $22 \mathrm{lbs} / 0.1 \mathrm{~s}$ or $220 \mathrm{lbs} / \mathrm{s}(997.9 \mathrm{~N} / \mathrm{s})$.

The sensor's resistance to fatigue was measured by cyclically loading to $30 \mathrm{lbs}(136.1 \mathrm{~N})$ for 1000 cycles at $1 \mathrm{~Hz}$ under displacement control (Fig. 16). The mean difference between sensor and load cell output was $-0.3 \pm 0.7 \mathrm{lbs}(-1.4 \pm 3.2 \mathrm{~N})$ with a $95 \%$ confidence interval between -1.8 and $1.1 \mathrm{lbs}(-8.2$ and
$4.9 \mathrm{~N})$. There is no time dependence in the difference between load cell and sensor outputs over the length of the test (linear regression $R^{2}=0.09$. Calibration curves were generated before and after fatigue and no significant difference was observed. The load cell's calibration also demonstrated no change after fatigue.

For clinical application, the sensor's response to materials of different stiffness was evaluated. In this work, two materials were chosen to mimic tissues that may be encountered in a clinical setting. The sensor was calibrated with no pad (between two rigid surfaces), between two soft, 1-cm-thick pads [modulus of elasticity $(E)=290 \mathrm{kPa}$ ], and between two stiffer 1-cm-thick pads $(E=400 \mathrm{kPa})$ (Fig. 17). The general shape for all three calibrations was similar but presented notable differences. The toe regions for the padded calibrations were significantly larger than the unpadded calibration. Also, sensitivity at low forces was approximately twice as high for the unpadded calibration than for both padded. Because of this, the overall loss at $40 \mathrm{lbs}(181.4 \mathrm{~N})$ was more than twice as high for the unpadded calibration. The calibration for the stiffer pad was repeatedly bimodal with an uncharacteristic linear region at $\sim 9 \mathrm{lbs}$ $(40.8 \mathrm{~N})$. The sensitivity for the softer padded test fell to zero at $30 \mathrm{lbs}(136.1 \mathrm{~N})$, representing its total operating range. The difference between padded and unpadded calibrations can be mostly attributed to the contact between top and bottom pads beyond the footprint of the sensor such that some load was transferred through the pads instead of exclusively through the center of the sensor.

One shortcoming of other identified sensors is their temperature dependence. In a clinical application, the sensor may be exposed to temperatures between $25^{\circ} \mathrm{C}$ (room temperature) and $37^{\circ} \mathrm{C}$ (body temperature). In other applications, the range may be higher. To test this sensor's temperature response, the sensor was heated with a heat gun and the temperature monitored from $23^{\circ} \mathrm{C}$ to $80^{\circ} \mathrm{C}$. With no force applied, the sensor showed no temperature response until the fiber began to curl above $80^{\circ} \mathrm{C}$. However, when loaded in displacement control to $30 \mathrm{lbs}(136.1 \mathrm{~N})$, the sensor's output decreased linearly after $35^{\circ} \mathrm{C}$ (Fig. 18). At $80^{\circ} \mathrm{C}$, the sensor's voltage output was decreased by $10 \%$, producing a $20 \mathrm{lbs}(90.7 \mathrm{~N})$ decrease because of its nonlinear sensitivity. When inspected manually, it was apparent that the plastic deforming plates had softened significantly which induced less bending in the fiber. If the elastomer had softened at all, its effect was masked by the decreased sensitivity caused by the softened plastic. For clinical application, the difference in temperature between normal body $\left(37^{\circ} \mathrm{C}\right)$ and



Fig. 15 Dynamic response and accuracy of sensor when loaded to 1/2 total range. 




Fig. 16 Fatigue resistance of the sensor as demonstrated by the difference between load cell and sensor peak outputs for 1000 cycles to $30 \mathrm{lbs}$ at $1 \mathrm{~Hz}$. The red bars indicate 1 standard deviation from the average difference (black).



Fig. 17 Tissue stiffness dependence on sensor calibration curves.

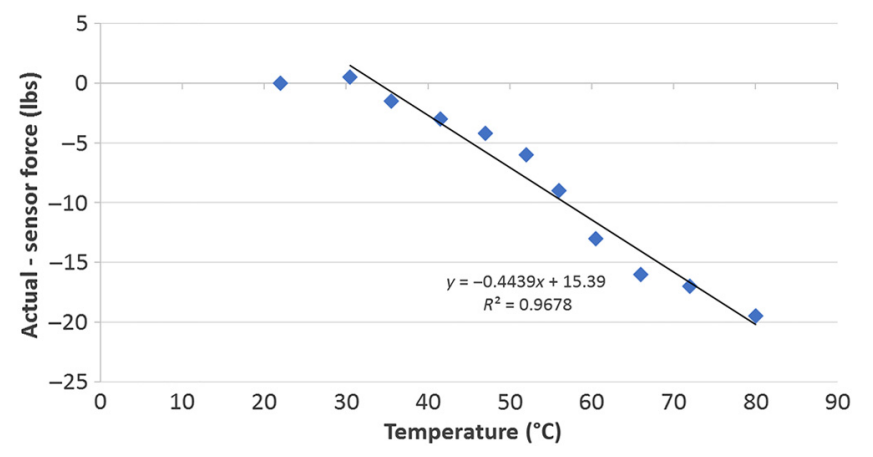

Fig. 18 The sensor's temperature response when loaded to $30 \mathrm{lbs}$. Linear regression excludes the first point $\left(23^{\circ} \mathrm{C}\right)$.

fever (say $40^{\circ} \mathrm{C}$ ) would have only a $4 \%$ impact on force measurement accuracy even if the sensor was fully heated to body temperature. In practice, it is not likely to reach the full internal body temperature of the patient and is likely to be closer to $30^{\circ} \mathrm{C}$ at which there is no difference in sensor performance from room temperature.
The resolution of force measurements is dependent on the sensitivity of the sensor at any given force. Analog-to-digital conversion is performed over 0 to $5 \mathrm{~V}$ with 14-bit resolution, providing $0.00031 \mathrm{~V} / \mathrm{pt}$. According to the average sensitivities measured, at $0 \mathrm{lbs}$, the sensitivity is $0.07 \mathrm{~V} / \mathrm{lb}(0.015 \mathrm{~V} / \mathrm{N})$, which, when divided, gives a resolution of $0.0044 \mathrm{lbs}(0.019$ $\mathrm{N})$. The highest resolution is coincident with highest sensitivity (at $\approx 2 \mathrm{lbs}$ or $9.07 \mathrm{~N})$ and is calculated to be $0.0017 \mathrm{lbs}(0.0077$ $\mathrm{N})$. As the sensitivity decreases closer to the range of the sensor, its resolution also decreases so that at $40 \mathrm{lbs}(181.4 \mathrm{~N})$, the resolution may be larger than $0.1 \mathrm{lbs}(0.45 \mathrm{~N})$. The smallest measureable force is $0.02 \mathrm{lbs}(0.09 \mathrm{~N})$ (measured by the sensor, not the load cell). This source of error is practically small compared to other sources of error. Other noticeable noise sometimes the sensor does pick up from is heartbeat if the sensor is placed directly over a major vessel, but the effect is relatively low (measured at $<0.2 \mathrm{lbs}$ worst case).

\subsection{Clinical Trial}

A longitudinal clinical trial was conducted at the University of Minnesota to investigate the effect of repeated force measurements performed on patients during rehabilitation following anterior cruciate ligament (ACL) reconstruction surgery (Fig. 19). Fifty-two patients were enrolled with mixed sex and ages between 18 and 50 years. These patients were randomized into treatment and nontreatment groups and were evaluated weekly during normal assessments. Over the duration of their recovery, active and passive range of motion (ROM), joint laxity, and quadriceps strength were measured and recorded (Fig. 19). The treatment group was evaluated quantitatively with the force-sensing glove system presented in this work, and the nontreatment group was evaluated with the current standard of care. Three physical therapists concurrently treated mixed groups of treatment and nontreatment patients for the 5-week duration reported here. The use of force feedback was the only experimental variable tested in this study.

Passive flexion and extension knee joint ROM was tracked for all patients during the first 5 weeks of treatment. This period of time is critical in determining the ultimate recovery of the 

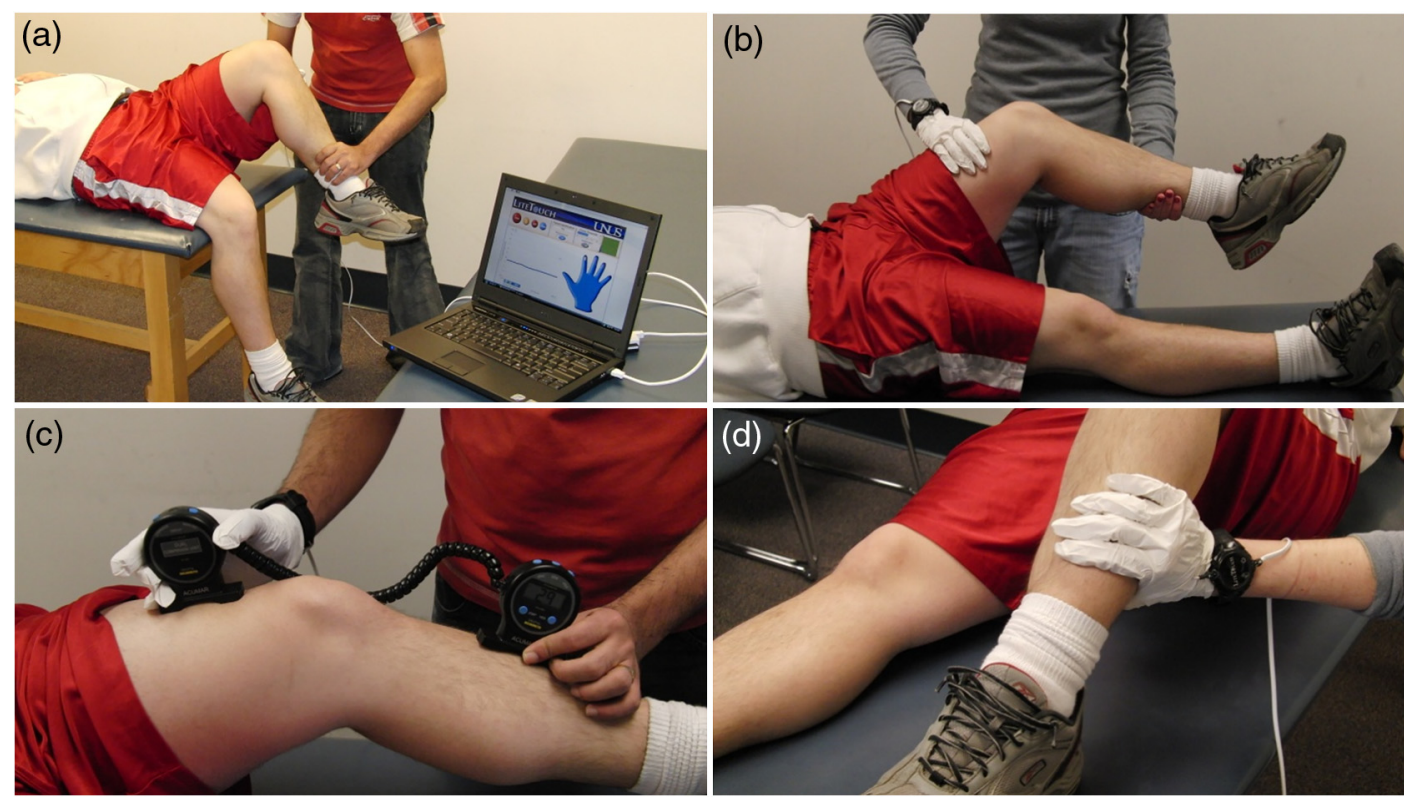

Fig. 19 Demonstration of therapeutic examination and measurements: (a) demonstrates the anterior drawer test, (b) illustrates the passive extension ROM test, (c) shows the inclinometer ROM measurement, and (d) depicts the quadriceps force measurement test.

joint after surgery. For the treatment group, the angular ROM of the joint was measured under $15 \mathrm{lbs}(68.0 \mathrm{~N})$ of applied force at midshaft of the femur for extension and midshaft of the tibia for flexion. ROM was reported by a standard inclinometer device and recorded in the patients' files. The nontreatment group underwent identical procedures but without any force feedback to indicate actual applied load. The measured angles are reported as a percent of ROM from the healthy contralateral knee.

Both groups show steady improvement in ROM over time. After week 1, the treatment group demonstrated better recovery of ROM than the nontreatment group in both extension measurements and after week 2 the same was true for flexion. After 5 weeks, the treatment group had recovered to $98.0 \%$ normal extension ROM and $91.2 \%$ normal flexion ROM compared to $91.4 \%$ and $84.3 \%$, respectively, for the nontreatment group (Fig. 20). Of the total 260 planned sessions, 10 data points were not collected because of missed appointments.

ACL laxity was measured with the glove during a Lashman test and with the KT-1000, the standard joint laxity measurement device. This head-to-head comparison served as a method to evaluate the glove's accuracy and utility when compared against the current state-of-the-art. The Lashman test is an anterior drawer test that measures the laxity in the knee joint by applying force to the back of the tibia and quadriceps. It is normally performed without force control but in this study, $15 \mathrm{lbs}$ were applied as measured by the glove to allow for evaluation over time and comparison to the KT-1000. Again, measurements were referenced to the contralateral knee. There appears to be no difference in the laxity measurements between the


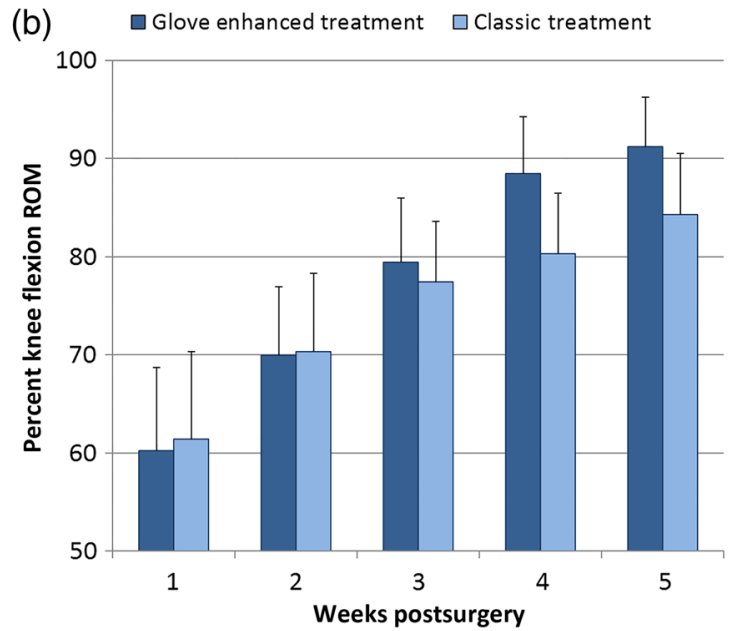

Fig. 20 Passive ROM of the knee during manipulation by a physical therapist. (a) Extension and (b) flexion ROM are reported as a percent of healthy (contralateral) ROM such that $100 \%$ represents full recovery. 




Fig. 21 Joint laxity measurement comparison between the gloveenhanced Lashman test and the KT-1000 measurement device. The force-sensing glove provided force feedback to control the forces applied during this test.

Lashman scoring system with force feedback from the glove and the KT-1000 score (Fig. 21). The data demonstrate similar means and standard deviations during the 6-week evaluation period.

Quadriceps muscle strength was measured every week to evaluate strength recovery postsurgery. Current strength measurement is scored relative to the contralateral muscle on a 0 to 5 scale in which the definition of every point is qualitative. The rubric is shown in Table 1. Muscle strength was quantified in the treatment group using the force-sensing glove. Figure 22 shows an inverse relationship between the muscle strength difference

Table 1 Scoring rubric for manual muscle strength testing.

\begin{tabular}{ll} 
Score & \multicolumn{1}{c}{ Definition } \\
\hline 5 & Normal strength \\
$5-$ & Barely detectable weakness \\
$4+$ & $\begin{array}{l}\text { Same as grade } 4, \text { but muscle holds the joint against } \\
\text { moderate to maximal resistance }\end{array}$ \\
4 & $\begin{array}{l}\text { Muscle holds the joint against a combination of } \\
\text { gravity and moderate resistance }\end{array}$ \\
$4-$ & $\begin{array}{l}\text { Same as grade } 4, \text { but muscle holds the joint only } \\
\text { against minimal resistance }\end{array}$ \\
$3+$ & $\begin{array}{l}\text { Muscle moves the joint fully against gravity, is } \\
\text { capable of transient resistance, but collapses abruptly }\end{array}$ \\
3 & $\begin{array}{l}\text { Muscle cannot hold the joint against resistance but } \\
\text { moves the joint fully against gravity }\end{array}$ \\
$3-$ & $\begin{array}{l}\text { Muscle moves the joint against gravity but not through } \\
\text { full mechanical ROM }\end{array}$ \\
2 & $\begin{array}{l}\text { Muscle moves the joint when gravity is eliminated } \\
1\end{array}$ \\
\hline
\end{tabular}



Fig. 22 Quadriceps strength measured with the glove ( $y$-axis) and scored on a 0 to 5 scale ( $x$-axis) relative to contralateral measurements. The week number is labeled for every point.

(measured by the glove) and the manual score, demonstrating good correlation between manual and glove strength measurement. Notably, however, the measurement variation found in the glove data is smaller than the manual scoring data. For instance, at week 2, average strength for all 26 patients is $\sim 16 \mathrm{~N}$ less than normal with a single standard deviation interval between 12 and $21 \mathrm{~N}$. In the manual scoring system, a single standard deviation away from average represents the difference between not being able to move against gravity and normal strength.

\section{Conclusion}

Theoretical calculations based on finite-element analysis and empirically derived bendloss formulas have been performed and show strong agreement with sensor characteristics. Because the equations used in this work contain condensed variables from unknown terms, the relationship is innately qualitative and is useful only for validation of the sensor's relative, not absolute behavior. The derived loss equation also allows performance predictions to be made when altering geometric parameters. Notably, it has been shown mathematically that increasing the pitch increases the range while decreasing sensitivity. The specific teeth geometry used in this work has proved to produce repeatable and reversible losses up to $89 \%$, providing high sensitivity over a large dynamic range. Under loading over $100 \mathrm{lbs}(453.6 \mathrm{~N})$, which is far outside the expected clinical applications, the fiber itself is protected from plastic deformation. Clinical criteria for range, sensitivity, and resolution have been exceeded. Notably, the sensor is sufficiently sensitive to detect the pulse from a wrist or carotid artery while robust enough to measure loads greater than $40 \mathrm{lbs}(181.4 \mathrm{~N})$. Fatigue over more than 1000 cycles has not been observed, indicating that a single sensor will continue providing highly repeatable force measurement for the duration of any clinical application. Because only a small volume of plastic, elastomer, and fiber are consumed in each sensor, the cost is sufficiently low for the gloves to be fully disposable.

Some shortcomings have been identified in this sensor, however. Current fabrication methodology produces inconsistent sensors due to the preloading protocol performed in load control. Future techniques should preload the fiber in displacement 
control during curing to more repeatably produce sensors. Accuracy to within $0.25 \mathrm{lbs}(1 \mathrm{~N})$ has been demonstrated at intermediate loading rates but is generally poorer at slower or faster rates (worse than $1.0 \mathrm{lbs}$ or $4.54 \mathrm{~N}$ ). Particularly, significant creep occurs when loaded for long durations. However, no other sensors of similar profile and footprint have been identified that demonstrate better dynamic fidelity. The current sensor is stable in temperatures up to $35^{\circ} \mathrm{C}$ but future sensors should be fabricated with a more temperature resistant plastic to increase its operating range to at least $40^{\circ} \mathrm{C}$.

For accurate clinical measurements, attention must be given to the stiffness of the tissue under loading. As expected, higher sensitivity has been demonstrated when tested between two rigid surfaces than more flexible materials. Calibration must be performed with tissue-like pads to mimic realistic loading conditions to obtain maximally accurate force measurement.

In this work, most sensor performance experiments were performed manually on a machine press with a calibrated load cell in series with the sensor. Although the forces measured and reported are accurate, significant noise was also introduced into the system because of the imprecise control of manual loading. Care was taken to eliminate human interaction but in some cases, including hysteresis measurements and cyclic loading, reliable and quantitative results were unobtainable. In future experimentation, a computer-controlled loading test machine should be used.

The clinical trial results presented in this work have demonstrated efficacy in improving patient recovery after ACL reconstruction surgery, validated the data collected against a standard quantitative clinical tool, and shown utility in decreasing variability in muscle measurement. This trial is ongoing and will continue to evaluate up to 120 patients over 12 weeks, whereby generating a significant body of data and a basis for making statistical judgments. Until then, statistical conclusions cannot be made from these results. However, it does appear that the glove has improved patient ROM recovery after just 2 weeks. Because the effect of the glove is not directly responsible for this improvement, a hypothesis must be made to account for this apparent success. It has been noted by the physical therapists that having access to force feedback in real time has allowed them to better track individual progress and provide better instructions and accountability for patient stretching and exercises during the week. This improved self-therapy has led to better outcomes. Another possible success mode is added motivation given to the patients upon observing their own performance quantitatively.

In conclusion, this force-sensing clinical glove represents a standard for quantifying clinical forces and establishes an unprecedented method for universally measuring and recording direct clinical force application. Unlike other measurement technologies currently available, this glove system measures direct loading between clinician and patient, minimally affects a clinician's normal function, and is fully disposable. Because of the system's low profile and simplicity, it may be readily adopted into clinical practice where a large body of foundational knowledge may be generated and shared among peers. Data from large-scale studies may become accessible and meaningful to all musculoskeletal clinicians with this device who may use published data to evaluate individual patients. The present work has demonstrated that the sensor and glove system have met all criteria established for clinical use and further developments will improve performance characteristics where needed.

\section{Disclosures}

The authors have no relevant financial interests in this article and no potential conflicts of interest to disclose.

\section{References}

1. G. B. Andersson, "Epidemiological features of chronic low-back pain," Lancet 354(9178), 581-585 (1999).

2. K. P. Lee et al., "Neurologic complications following chiropractic manipulation: a survey of California neurologists," Neurology 45(6), 1213-1215 (1995).

3. P. Rizun et al., "Mechatronic design of haptic forceps for robotic surgery," Int. J. Med. Rob. Assisted Surg. 2(4), 341-349 (2006).

4. M. Lee, A. Moseley, and K. Refshauge, "Effect of feedback on learning a vertebral joint mobilization skill," Phys. Ther. 70(2), 97-102 (1990).

5. K. J. McQuade and A. M. Murthi, "Anterior glenohumeral force/ translation behavior with and without rotator cuff contraction during clinical stability testing," Clin. Biomech. 19, 10-15 (2004).

6. R. A. Hintermeister et al., "Quantification of elastic resistance knee rehabilitation exercises," J. Orthop. Sports Phys. Ther. 28(1), 40-50 (1998).

7. U. Levin, L. Nilsson-Wikmar, and C. H. Stenström, "Variability within and between evaluations of sacroiliac pain with the use of distraction testing," J. Manipulative Physiol. Ther. 28(9), 688-695 (2005).

8. S. J. Snodgrass, D. A. Rivett, and V. J. Robertson, "Manual forces applied during posterior-to-anterior spinal mobilization: a review of the evidence," J. Manipulative Physiol. Ther. 29(4), 316-329 (2006).

9. G. Waddington, J. Diong, and R. Adams, "Development of a hand dynamometer for the control of manually applied forces," J. Manipulative Physiol. Ther. 29(4), 297-304 (2007).

10. G. Burdea et al., "Computerized hand diagnostic/rehabilitation system using a force feedback glove," Stud. Health Technol. Inf. 39, 141-150 (1997).

11. A. Nikonovas et al., "The application of force-sensing resistor sensors for measuring forces developed by the human hand," Proc. Inst. Mech. Eng. Part H 218, 121-126 (2004).

12. C. Pylatuik et al., "Distribution of grip force in three different functional prehension patterns," J. Med. Eng. Technol. 30, 176-182 (2006).

13. K. N. Tarchanidis and J. N. Lygouras, "Data glove with a force sensor," IEEE Trans. Instrum. Meas. 52, 984-989 (2003).

14. M. C. F. Castro and A. Cliquet, "A low-cost instrumented glove for monitoring forces during object manipulation," IEEE Trans. Rehabil. Eng. 5, 140-147 (1997)

15. A. Vijayan et al., "An optical fiber weighing sensor based on bending," Meas. Sci. Technol. 19, 105302 (2008).

16. J. W. Berthold, "Historical review of microbend fiber-optic sensors," J. Lightwave Technol. 13, 1193-1199 (1995).

17. A. Kulkarni et al., "Optically activated novel force sensor calibrated as weighing balance," Microwave Opt. Technol. Lett. 45, 300-303 (2005).

18. M. Linec and D. Donlagic, "A plastic optical fiber microbend sensor used as a low-cost anti-squeeze detector," IEEE Sens. J. 7, 1262-1267 (2007).

19. S. F. Knowles et al., "Multiple microbending optical-fibre sensors for measurement of fuel quantity in aircraft fuel tanks," Sens. Acutators A: Phys. 68, 320-323 (1998).

20. N. K. Pandey and B. C. Yadav, "Fibre optic pressure sensor and monitoring of structural defects," Opt. Appl. 37, 57-63 (2007).

21. W.-C. Wang et al., "A shear and plantar pressure sensor based on fiberoptic bend loss," J. Rehabil. Res. Dev. 42(3), 315-326 (2005).

22. W.-C. Wang et al., "Developments of a microfabricated optical bend loss sensor for distributive pressure measurement," IEEE Trans. Biomed. Eng. 55(2), 614-625 (2008).

23. D. Linders, D. Nuckley, and W.-C. Wang, "Optical clinical force sensing glove," in 16th SPIE NDE Health Monitoring and Diagnostics, San Diego, California (2009).

24. D. Linders, W.-C. Wang, and D. Nuckley, "Designing an optical bendloss sensor for clinical force measurement," J. Med. Devices 3(2), 027522 (2009). 
Wang, Linders, and Nuckley: Development of a disposable force-sensing glove for clinicians and demonstration...

25. S. Tsao and W. Cheng, "Simplified formula of bending loss for optical fiber sensors," Fiber Integr. Opt. 21, 333-344 (2002).

26. A. A. P. Boechat et al., "Bend loss in large core multimode optical fiber beam delivery systems," Appl. Opt. 30, 321-327 (1991).

Wei-Chih Wang is currently an associate professor in the Department of Power Mechanical Engineering, Institute of Nanoengineering and
Microsystems, National Tsinghua University. His research interests are in the area of developing polymer-based microsensors and actuators for industrial and biomedical applications. More recently, his work has been expanded to $\mathrm{THz}, \mathrm{IR}$, and visible band 3-D advance electromagnetic material and structure study.

Biographies for the other authors are not available. 